\title{
Correlation between karyotype and quantitative immunophenotype in acute myelogenous leukemia with $t(8 ; 21)$
}

Haytham Khoury, ${ }^{1}$, Bakul I Dalal ${ }^{2}$, Stephen H Nantel ${ }^{3}$, Douglas E Horsman ${ }^{4}$, Julye C Lavoie ${ }^{3}$, John D Shepherd ${ }^{3}$, Donna E Hogge ${ }^{3}$, Cynthia L Toze ${ }^{3}$, Kevin W Song ${ }^{3}$, Donna L Forrest ${ }^{3}$, Heather J Sutherland ${ }^{3}$ and Thomas J Nevill ${ }^{3}$

\author{
${ }^{1}$ Department of Cellular and Molecular Biology, Princess Margaret Hospital, Toronto, Ontario, Canada; \\ ${ }^{2}$ Department of Pathology and Laboratory Medicine, Vancouver Hospital and Health Sciences Center, \\ Vancouver, Canada; ${ }^{3}$ Leukemia/BMT Program of British Columbia, BC, Canada and ${ }^{4}$ Department of \\ Pathology and Laboratory Medicine, British Columbia Cancer Agency, BC, Canada
}

\begin{abstract}
Acute myelogenous leukemia with $t(8 ; 21)$ is a distinct clinicopathologic entity in which the malignant myeloblasts display a characteristic pattern of surface antigen expression. Quantitative analysis of surface marker expression in patients with this chromosomal abnormality compared to acute myelogenous leukemia patients with a different karyotype has not been reported. From 305 consecutive newly diagnosed acute myelogenous leukemia patients underwent immunophenotyping and cytogenetic analysis at our center; 16 patients $(5.2 \%)$ had a t(8;21). Fluorescence intensity values were obtained, using a set of reference microbeads, by conversion of mean channel fluorescence to molecular equivalent of soluble fluorochrome. Patients with $t(8 ; 21)$ displayed higher levels of CD34, HLA-DR and MPO expression $(P<0.001$ for each) and lower levels of CD13 $(P=0.03)$ and CD33 $(P=0.02)$ expression. In order to study the sensitivity, specificity and predictive value of these markers, molecular equivalent of soluble fluorochrome thresholds were statistically determined. The statistically established threshold for each of the individual markers (CD34 $>60.5 \times 10^{3}, \mathrm{HLA}-\mathrm{DR}>176.1 \times 10^{3}$, MPO $>735.1 \times 10^{3}$, CD13 $<24.3 \times 10^{3}$ and CD33 $<17.3 \times 10^{3}$ ) had a sensitivity of $100 \%$, a specificity of $62-92 \%$ and a positive predictive value of 7-45\%. In multivariate analysis, two quantitative patterns (CD34 $>60.5 \times 10^{3}$ and MPO $>176.1 \times 10^{3} ;$ CD33 $<17.3 \times 10^{3}$ and MPO $>176.1 \times 10^{3}$ ) had a sensitivity, specificity and positive predictive value of $100 \%$. These aberrant phenotypic patterns might help identify patients with $t(8 ; 21)$ at diagnosis and could be useful in minimal residual disease monitoring.

Modern Pathology (2004) 17, 1211-1216, advance online publication, 4 June 2004; doi:10.1038/modpathol.3800168
\end{abstract}

Keywords: AML; t(8;21); quantitative immunophenotype

In patients with acute myelogenous leukemia (AML), $\mathrm{t}(8 ; 21)(\mathrm{q} 22 ; \mathrm{q} 22)$ is a relatively frequent structural cytogenetic abnormality. This chromosomal translocation results in an in-frame fusion between the first 5 exons of the AML1 gene and essentially all of the ETO gene producing a chimeric protein. ${ }^{1}$ This protein, AML1-ETO, retains the ability of AML1 to heterodimerize with $\operatorname{CBF} \beta$ and to bind DNA as well as allowing ETO to interact with the N-Co-R/Sin3/HDAC complex. ${ }^{2}$ This results in its acting as a negative dominant inhibitor of

Correspondence: Dr H Khoury, MD, Princess Margaret Hospital, Department of Cellular and Molecular Biology, Room 9-111, 610 University Ave., Toronto, Ontario, Canada M5G 2M9.

E-mail: khaytham@uhnres.utoronto.ca

Received 3 December 2003; revised 22 March 2004; accepted 23 March 2004; published online 4 June 2004 wild-type AML1. ${ }^{3}$ The creation of AML1-ETO is necessary, but not sufficient, for leukemogenesis in AML with $\mathrm{t}(8 ; 21){ }^{4}$

The recently developed WHO classification of hematologic malignancies recognizes AML with $\mathrm{t}(8 ; 21)$ as a distinct clinicopathologic entity. ${ }^{5}$ This form of AML is found more frequently in children and young adults ${ }^{6}$ and patients are predisposed to extramedullary localization. ${ }^{7} \mathrm{Com}-$ plete remission rates and long-term event-free survival are high, particularly when treatment incorporates high-dose cytosine arabinoside. ${ }^{8}$ Histopathology characteristically demonstrates frequent type III myeloblasts, Auer rods, cytoplasmic vacuolization, pseudo-Chediak-Higashi granules, pink globules or salmon-colored cytoplasm, eosinophilia with abnormal granulation and granulocytic dysplasia. ${ }^{6,9,10}$ 
Characteristic qualitative flow cytometry features have also been described in $\mathrm{t}(8 ; 21)$ disease including a higher incidence of CD34, TdT, CD19 and CD56 expression as well as a lower incidence of CD33 expression. ${ }^{11-13}$ We have shown that combined CD34, CD19 and CD56 positivity in AML patients has a $67 \%$ sensitivity, $100 \%$ specificity and a positive predictive value of $100 \%$ for the presence of $t(8 ; 21) .{ }^{14}$ Despite support for the existence of a unique immunophenotype in AML with $t(8 ; 21)$, quantitative immunophenotyping in this form of AML has rarely been reported. ${ }^{13}$ Recent reports have suggested that quantitative immunophenotyping may enhance the diagnosis of leukemias with specific cytogenetic abnormalities ${ }^{15-17}$ and help define aberrant immunophenotyping patterns that could be useful for monitoring minimal residual disease. ${ }^{17-19}$ It has also been suggested that quantitative immunophenotyping can contribute to our understanding of the pathogenesis of acute leukemia. ${ }^{13,15,20}$ We undertook this prospective study of quantitative immunophenotyping to define whether there is a distinctive pattern in patients with AML and $t(8 ; 21)$.

\section{Materials and methods}

\section{Patient Selection}

Between January 1998 and June 2003, 327 adults with AML had a bone marrow sample referred to the Vancouver General Hospital for flow cytometry assessment. The diagnosis of AML was based upon standard FAB criteria. ${ }^{21}$ We excluded from the analysis, 22 patients who did not have a bone marrow karyotype available from diagnosis because either cytogenetics were not performed (14 patients) or no analyzable metaphases were present (eight patients). Of the remaining 305 patients, 16 patients $(5.2 \%)$ had evidence of $\mathrm{t}(8 ; 21)$ on standard metaphase analysis. Quantitative immunophenotyping data from the 13 patients with $t(8 ; 21)$ was compared to the 292 patients lacking this abnormality.

\section{Immunophenotyping}

All immunophenotyping was performed on fresh bone marrow samples obtained at the time of diagnosis. The specimen was processed either by a whole blood lysis technique using hypotonic solution or by isolating the blast cells through density centrifugation and washing three times in PBS. The cells were stained by direct immunofluorescence technique using a panel of monoclonal antibodies (moAb) conjugated to either FITC, PE or PerCP. The routine AML moAb panel included CD2, CD3, CD4, CD7, CD8, CD10, CD11b, CD11c, CD13, CD14, CD19, CD33, CD34, CD45 and CD56 (all Becton Dickinson, San Jose, CA, USA), TdT (Supertechs Inc., Rockville, MD, USA) and myeloperoxidase (MPO) from Immunotech (Marseille, France). Briefly, antibodies were mixed at appropriate dilutions with $1 \times 10^{5}$ cells, incubated for $30 \mathrm{~min}$ at $-4^{\circ} \mathrm{C}$, washed in PBS and postfixed in $1 \%$ paraformaldehyde. Immunophenotyping analysis was performed on either a Coulter Epics Profile II (Coulter Inc, Hialiah, FL, USA) or a Facscaliber (Becton Dickinson, San Jose, CA, USA) flow cytometer. The laser alignment was verified daily using DNA-Check and fluorescence was standardized using Standard-Brite beads (Coulter Inc, Hialiah, FL, USA). The cells were gated using CD45 vs side scatter gating method with the specimen being considered positive for a surface marker if $\geq 20 \%$ of cells expressed the marker being tested. Coexpression of markers was assessed by a direct method in which different antibodies were added to a single sample tube.

\section{Quantitative Analysis}

To quantify the antigen fluorescence intensity, the mean channel fluorescence (MCF) values were converted into a molecular equivalent of soluble fluorochrome (MESF) using Quantum Simply Cellular $^{\mathrm{TM}}$ microbead kit (Flow Cytometry Standards Corporation, San Juan, Puerto Rico). The kit contained beads of five different fluorescence intensities producing five peaks on the flow cytometry histogram; one peak was a reference blank and the four others had predetermined MESF values. Instrument calibration for measurement of fluorescence intensity in MESF units was performed on a daily basis. The fluorescence beads were analyzed with the same instrument setting as those used for the analysis of the patient samples. After running the microbeads in the flow cytometer, a calibration plot to convert MCF to MESF was established. A linear regression equation correlating the channel number with a specific MESF value was calculated using Quickcal $^{\text {TM }}$ software (Flow Cytometry Standards Corporation, San Juan, Puerto Rico).

\section{Cytogenetic Analysis}

Chromosome analysis was performed on bone marrow aspirates using standard 24-h culture and harvest procedures followed by G-banding, as previously reported. The karyotypes were reported according to published ISCN guidelines. ${ }^{22}$

\section{Statistical Analysis}

The mean and median MESF of each surface marker for patients with and without $\mathrm{t}(8 ; 21)$ was calculated along with the standard deviation (s.d. ) and 95\% confidence interval (CI). For the antigens that showed a difference in intensity between AML with $\mathrm{t}(8 ; 21)$ and other AML patients, MESF thresholds that helped to distinguish between the two groups were devised. For this purpose, the classification 
and regression tree (CART) analysis was used. ${ }^{23}$ Comparison of qualitative variables was performed using a Fisher's exact test. Student's $t$-test was used to compare quantitative variables after checking the distribution normality for each variable, using the GraphPAD Prism ${ }^{\mathrm{TM}}$ software. Sensitivity, specificity and positive predictive value were calculated according to the standard formulae. ${ }^{24}$ Multivariate analysis examining different marker combinations was performed using a logistic regression model (SPSS program).

\section{Results}

\section{Qualitative Immunophenotyping}

Qualitative immunophenotyping results for the patient cohort are summarized in Table 1. Patients with $\mathrm{t}(8 ; 21)$ showed a higher incidence of CD34 $(P=0.02)$, TdT $(P=0.003)$, CD19 $(P<0.001)$ and CD56 $(P<0.001)$ positivity and a lower incidence of CD33 positivity $(P=0.002)$ compared to AML patients with other karyotypes.

\section{Quantitative Immunophenotyping}

Quantitative immunophenotyping results are summarized in Table 1. AML patients with $t(8 ; 21)$ demonstrated a higher intensity of CD34 $(P<0.001)$, HLA-DR $(P<0.001)$ and $\mathrm{MPO}$ $(P<0.001)$ expression compared to patients lacking $\mathrm{t}(8 ; 21)$ (Figure 1$)$. In addition, $\mathrm{t}(8 ; 21)$ patients had a lower CD13 $(P=0.025)$ and CD33 $(P=0.02)$ intensity than other patients (Figure 1).

\section{MESF Thresholds and Predictive Values}

MESF thresholds that could distinguish between AML patients with $\mathrm{t}(8 ; 21)$ and other AML patients were established by using CART analysis. These thresholds were set at $\mathrm{MESF}>60.5$ for CD34, $>176.1$ for HLA-DR, > 735.1 for MPO, <24.3 for CD13 and $<17.3$ for CD33 (Table 2). The sensitivity, specificity and predictive values for individual surface marker thresholds are shown in Table 2 . Each of these marker thresholds had a sensitivity of $100 \%$ with specificities of $62-92 \%$. The predictive values for different quantitative marker threshold combinations are shown in Table 3. Two combinations (CD34 and MPO; CD33 and MPO) had both a sensitivity and a positive predictive value of $100 \%$.

\section{Discussion}

The surface antigens on hematopoietic cells that are studied by flow cytometry are differentiation

Table 1 Comparison of antigen quantitation between acute myelogenous leukemia with $(n=16)$ and without $(n=289) \mathrm{t}(8 ; 21)$

\begin{tabular}{|c|c|c|c|}
\hline Surface marker & Cytogenetics & Positive patients (\%) & Mean $M E S F \pm s . d .\left(\times 10^{3}\right)$ \\
\hline \multirow[t]{3}{*}{ CD34 } & $t(8 ; 21)$ & 100 & $136 \pm 20.6$ \\
\hline & Others & 70 & $52.7 \pm 38.9$ \\
\hline & & $P=0.02$ & $P<0.001$ \\
\hline \multirow[t]{3}{*}{ HLA-DR } & $\mathrm{t}(8 ; 21)$ & 100 & $349.8 \pm 44.6$ \\
\hline & Others & 82 & $116.2 \pm 140.5$ \\
\hline & & $P=0.13$ & $P<\overline{0.001}$ \\
\hline \multirow[t]{3}{*}{$\mathrm{TdT}$} & $t(8 ; 21)$ & 67 & $48.1 \pm 30.3$ \\
\hline & Others & 25 & $71.7 \pm 78.5$ \\
\hline & & $P=0.003$ & $P=0.56$ \\
\hline \multirow{3}{*}{ CD13 } & $\mathrm{t}(8 ; 21)$ & 100 & $15.3 \pm 2.4$ \\
\hline & Others & 95 & $40.3 \pm 31.2$ \\
\hline & & $P=1.0$ & $P=0.03$ \\
\hline \multirow[t]{3}{*}{ CD33 } & $t(8 ; 21)$ & 69 & $9.1 \pm 2.0$ \\
\hline & Others & 96 & $33.1 \pm 25.4$ \\
\hline & & $P=0.002$ & $P=\overline{0.02}$ \\
\hline \multirow[t]{3}{*}{ MPO } & $t(8 ; 21)$ & 100 & $1189.0 \pm 87.7$ \\
\hline & Others & 89 & $351.4 \pm 353.5$ \\
\hline & & $P=0.59$ & $P<0.001$ \\
\hline \multirow[t]{3}{*}{ CD15 } & $\mathrm{t}(8 ; 21)$ & 90 & $69.8 \pm 53.0$ \\
\hline & Others & 65 & $139 \pm 188.1$ \\
\hline & & $P=0.17$ & $P=\overline{0.56}$ \\
\hline \multirow[t]{3}{*}{ CD11c } & $t(8 ; 21)$ & 100 & $9.2 \pm 6.7$ \\
\hline & Others & 78 & $35.1 \pm 31.2$ \\
\hline & & $P=0.21$ & $P=0.19$ \\
\hline \multirow[t]{3}{*}{ CD19 } & $t(8 ; 21)$ & 85 & $12.4 \pm 9.0$ \\
\hline & Others & 9 & $18.8 \pm 30.6$ \\
\hline & & $P<0.001$ & $P=0.17$ \\
\hline \multirow{3}{*}{ CD56 } & $t(8 ; 21)$ & 75 & $39 \pm 16.2$ \\
\hline & Others & 19 & $25.2 \pm 16.3$ \\
\hline & & $P<0.001$ & $P=\overline{0.09}$ \\
\hline
\end{tabular}

s.d., standard deviation; MESF, molecular equivalent of soluble fluorochrome; TdT, terminal deoxynucleotidyl transferase; MPO, myeloperoxidase. 


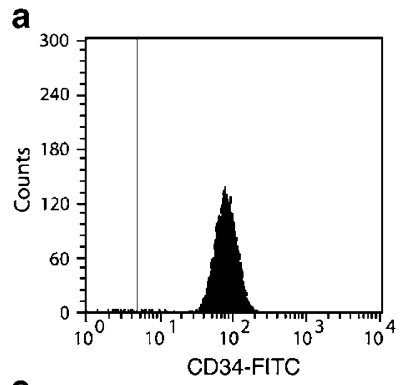

C
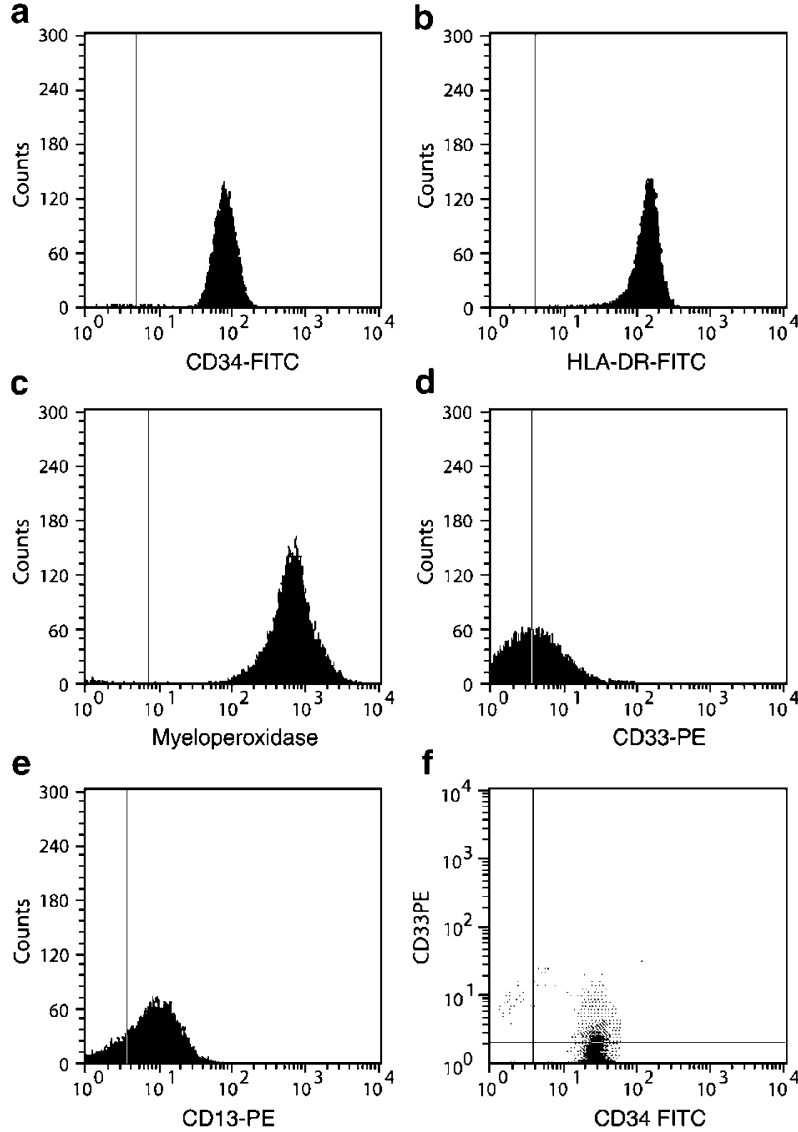

d

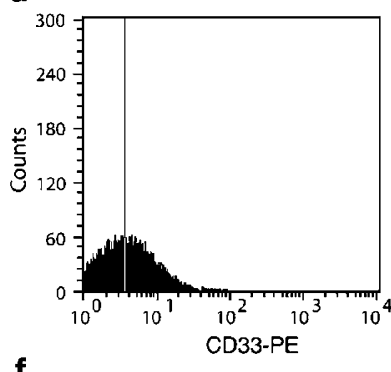

f

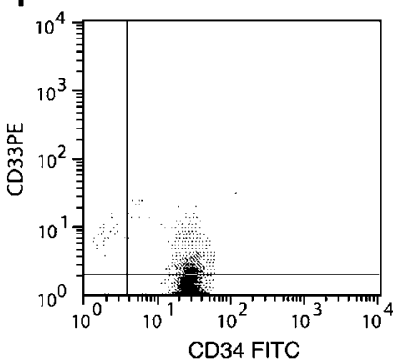

Figure 1 Quantitative immunophenotypic characteristics of AML with $\mathrm{t}(8 ; 21)$. High expression of CD34, HLA-DR and MPO (a-c). Low expression of CD33 and CD13 (d,e). Typical pattern of expression of CD34-FITC/CD33-PE showing high CD34 with partial and weak CD33 expression (f).

Table 2 Sensitivity, specificity and predictive values of individual surface marker MESF thresholds for patients with acute myelogenous leukemia with $\mathrm{t}(8 ; 21)$

\begin{tabular}{lcccc}
\hline $\begin{array}{l}\text { MESF threshold } \\
\left(\times 10^{3}\right)\end{array}$ & $\begin{array}{c}\text { Sensitivity } \\
(\%)\end{array}$ & $\begin{array}{c}\text { Specificity } \\
(\%)\end{array}$ & $\begin{array}{r}P P V \\
(\%)\end{array}$ & $\begin{array}{c}\text { NPV } \\
(\%)\end{array}$ \\
\hline CD34 $>60.5$ & 100 & 78 & 12 & 100 \\
HLA-DR $>176.1$ & 100 & 87 & 19 & 100 \\
MPO $>735.1$ & 100 & 92 & 45 & 100 \\
CD13 $<24.3$ & 100 & 62 & 7 & 100 \\
CD33 $<17.3$ & 100 & 65 & 8 & 100 \\
\hline
\end{tabular}

MESF, molecular equivalent of soluble fluorochrome; PPV, positive predictive value; NPV, negative predictive value; MPO, myeloperoxidase.

proteins that are regulated in a coordinated way in normal hematopoiesis. ${ }^{20}$ Leukemic hematopoiesis is characterized by asynchronous regulation of differentiation genes. ${ }^{20}$ The quantitation of antigen density is an important tool to identify characteristics of these differentiation abnormalities and thereby provide insight into the pathogenesis of specific leukemia subtypes. ${ }^{13,15}$ Furthermore, the determina-

Table 3 Sensitivity, specificity and predictive values of surface marker MESF threshold combinations for acute myelogenous leukemia with $\mathrm{t}(8 ; 21)$

\begin{tabular}{|c|c|c|c|c|}
\hline $\begin{array}{l}\text { MESF thresholds } \\
\left(\times 10^{3}\right)\end{array}$ & $\begin{array}{l}\text { Sensitivity } \\
(\%)\end{array}$ & $\begin{array}{c}\text { Specificity } \\
(\%)\end{array}$ & $\begin{array}{l}P P V \\
(\%)\end{array}$ & $N P V(\%)$ \\
\hline $\begin{array}{l}\mathrm{CD} 34>60.5, \\
\mathrm{MPO}>735.1\end{array}$ & 100 & 100 & 100 & 100 \\
\hline $\mathrm{CD} 33<17.3$ & & & & \\
\hline $\begin{array}{l}\mathrm{MPO}>735.1 \\
\mathrm{CD} 34>60.5\end{array}$ & 100 & 100 & 100 & 100 \\
\hline $\begin{array}{l}\text { HLA-DR }>176.1 \\
\text { CD34 > 60.5, }\end{array}$ & 100 & 99 & 81 & 100 \\
\hline $\begin{array}{l}\text { CD13<24.3 } \\
\text { CD33 }<17.3\end{array}$ & 100 & 99 & 76 & 100 \\
\hline $\begin{array}{l}\text { HLA-DR }>176.1 \\
\text { CD13<24.3, }\end{array}$ & 100 & 98 & 72 & 100 \\
\hline HLA-DR > 176.1 & 100 & 98 & 71 & 100 \\
\hline
\end{tabular}

MESF, molecular equivalent of soluble fluorochrome; PPV, positive predictive value; NPV, negative predictive value; MPO, myeloperoxidase.

tion of aberrant phenotypic patterns can also allow for minimal residual disease (MRD) monitoring. ${ }^{17,19}$

AML with $t(8 ; 21)$ is characterized by a unique molecular abnormality, the AML1-ETO fusion gene. It has been shown that AML1-ETO protein can regulate the transcription of many genes important for hematopoiesis including the M-CSF receptor, the G-CSF receptor and BCL2 (all upregulated) as well as GM-CSF, TCR subunits $(\alpha, \beta$ and $\delta)$, NP3 and MDR1 (all downregulated). ${ }^{25}$ In addition, the AML1ETO chimeric protein leads to dysplastic granulopoiesis in a knockin mice model. ${ }^{26}$ Dysplastic granulopoiesis, a high density of G-CSF and MCSF receptors and a low density of MDR1 have been noted in leukemic blasts with $\mathrm{t}(8 ; 21){ }^{10,27-29}$ Collectively, these data suggest that in this unique form of AML, the phenotypic features probably result from intrinsic molecular alterations. It would follow that AML with $\mathrm{t}(8 ; 21)$ could have a unique surface antigen expression pattern.

A correlation between the presence of $t(8 ; 21)$ and the qualitative expression of certain surface antigens on blast cells has previously been reported..$^{11-13}$ In these studies, it was found that AML with $t(8 ; 21)$ is characterized by a higher incidence of CD34, TdT, CD19 and CD56 positivity and a lower incidence of CD33 positivity. Our present study has confirmed these results and, in addition, provides a quantitative analysis of surface and cytoplasmic markers in this disease. To our knowledge, only one previous study has reported results of this type of analysis in AML with $\mathrm{t}(8 ; 21)$. Porwit-MacDonald et $a l^{13}$ found that AML with $\mathrm{t}(8 ; 21)$ is associated with high intensity CD34 expression. The current study undertook a more comprehensive analysis of quantitative antigen expression in AML with $\mathrm{t}(8 ; 21)$. It is now apparent that, in addition to CD34, this cytogenetic abnormality is associated with a higher intensity of HLA-DR and MPO expression as well as a lower intensity of CD13 and CD33 expression. 
The quantitative thresholds established in this study are not defined by arbitrary cut-offs, but are based on well-established statistical calculations allowing for broad applicability of the thresholds to a larger population of patients. Our analysis not only established the predictive values for the individual quantitative surface marker thresholds but also determined the predictive values for various marker combinations. Each of the individual marker thresholds for CD34, HLA-DR, MPO, CD13 and CD33 had 100\% sensitivity for the presence of $t(8 ; 21)$ although the positive predictive values were low. In fact, the MPO threshold had the highest positive predictive value at only $45 \%$. However, when the predictive values of different combinations of thresholds were analyzed, two patterns with $100 \%$ sensitivity, specificity and positive/negative predictive values emerged (CD34 and MPO; CD33 and MPO). We have previously reported a qualitative pattern of immunophenotyping $\left(\mathrm{CD} 19^{+}, \mathrm{CD} 56^{+}\right.$ and $\mathrm{CD}_{34}{ }^{+}$) with a sensitivity of $100 \%$ and a positive predictive value of $67 \% .{ }^{14}$ Although in our present series of patients this pattern had a sensitivity of only $62 \%$, it retained a positive predictive value of $100 \%$ (data not shown). These results suggest that quantitative immunophenotyping may be better than qualitative immunophenotyping at predicting which patients with AML have a $t(8 ; 21)$ karyotype.

Although the incidence of AML with cryptic $t(8 ; 21)$ is relatively low, ${ }^{30,31}$ the detection of these cases has significant clinical relevance. AML with $t(8 ; 21)$ has a favorable prognosis and is often curable with consolidation chemotherapy alone. ${ }^{8,32}$ Patients may have a cryptic $\mathrm{t}(8 ; 21)$ which can occur in patients with apparently normal (intermediate-risk) cytogenetics or in patients with complex (high-risk) karyotypes. ${ }^{6,9}$ Cytogenetics are frequently used to determine intensity of postremission therapy in AML and patients with either normal or high-risk karyotypes may be preferentially referred for allogenic bone marrow transplantation whereas goodrisk patients would not. In this regard, detection of a cryptic $t(8 ; 21)$ might result in the avoidance of more toxic therapies. While we did not perform molecular testing for an AML1-ETO fusion on patients who did not have $\mathrm{t}(8 ; 21)$, all had flow cytometry screening. The consistency of our immunophenotyping findings suggests that very few patients could have (if any) a cryptic $\mathrm{t}(8 ; 21)$ abnormality.

In normal hematopoiesis, progenitors expressing medium to high levels of CD34 antigen are characterized by absence or low expression of MPO. As the CD34 density decreases, the intensity of MPO increases. ${ }^{33} \mathrm{~A}$ correlation between MPO and CD33 expression has also been observed in normal hematopoietic development and MPO-expressing cells have significantly stronger CD33 expression. ${ }^{33}$ In this study, we defined at least two aberrant antigen intensity patterns: coexpression of CD34 $4^{\text {high }}$ and $\mathrm{MPO}^{\text {high }}$ and coexpression of $\mathrm{MPO}^{\text {high }}$ and

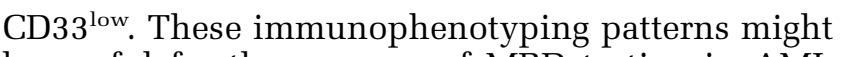
be useful for the purpose of MRD testing in AML patients with $\mathrm{t}(8 ; 21)$.

AML with $t(8 ; 21)$ is a form of leukemia with unique clinicopathologic features. Our results confirm the qualitative immunophenotyping characteristics previously reported for $\mathrm{t}(8 ; 21)$ patients and describes new quantitative immunophenotyping results that can help identify this underlying cytogenetic abnormality. This could prove useful in MRD monitoring in AML patients with $t(8 ; 21)$ but it remains to be seen whether flow cytometry will be helpful in determining induction therapy for AML patients.

\section{References}

1 Erickson PF, Robinson M, Owens G, et al. The ETO portion of acute myeloid leukemia $t(8 ; 21)$ fusion transcript encodes a highly evolutionarily conserved, putative transcription factor. Cancer Res 1994;54: 1782-1786.

2 Lutterbach B, Westendorf JJ, Linggi B, et al. ETO, a target of $\mathrm{t}(8 ; 21)$ in acute leukemia, interacts with the $\mathrm{N}$ CoR and mSin3 corepressors. Mol Cell Biol 1998; 18:7176-7184.

3 Meyers S, Lenny N, Hiebert SW. The $t(8 ; 21)$ fusion protein interfers with AML-1-B-dependant transcriptional activation. Mol Cell Biol 1995;15:1974-1982.

4 Yuan Y, Zhou L, Miamoto T, et al. AML-ETO expression is directly involved in the development of acute myeloid leukemia in the presence of additional mutations. Proc Natl Acad Sci USA 2001;98:1039810403.

5 Harris NL, Jaffe ES, Diebold J, et al. World Health Organization Classification of neoplastic diseases of the hematopoietic and lymphoid tissues. J Clin Oncol 1999;17:3835-3849.

6 Andrieu V, Radford-Weiss I, Troussard X, et al. Molecular detection of $t(8 ; 21) / A M L 1-E T O$ in AML M1/M2: correlation with cytogenetics, morphology and immunophenotype. $\mathrm{Br} J$ Haematol 1996;92: 855-865.

7 Byrd JC, Weiss RB. Recurrent granulocytic sarcoma. An unusual variation of acute myelogenous leukemia associated with 8;21 chromosomal translocation and blast expression of neural cell adhesion molecule. Cancer 1994;15:2107-2112.

8 Bloomfield CD, Lawrence D, Byrd JC, et al. Frequency of prolonged remission duration after high-dose cytarabine intensification in acute myeloid leukemia varies by cytogenetic subtype. Cancer Res 1998;58:41734179.

9 Nucifora G, Dickstein JI, Torbenson V, et al. Correlation between cell morphology and expression of the AML1/ ETO chimeric transcript in patients with acute myeloid leukemia without the $\mathrm{t}(8 ; 21)$. Leukemia 1994;8: 1533-1538.

10 Haferlach T, Bennett JM, Loffler $\mathrm{H}$, et al. Acute myeloid leukemia with translocation $(8 ; 21)$. Cytomorphology, dysplasia and prognostic factors in 41 cases. Leuk Lymphoma 1996;23:227-234.

11 Hurwitz CA, Raimondi SC, Head D, et al. Distinctive immunophenotypic features of $\mathrm{t}(8 ; 21)(\mathrm{q} 22 ; \mathrm{q} 22)$ acute 
myeloblastic leukemia in children. Blood 1992;80: 3182-3188.

12 Kita K, Nakase K, Miwa $\mathrm{H}$, et al. Phenotypical characteristics of acute myelocytic leukemia associated with $\mathrm{t}(8 ; 21)$ chromosomal abnormality: frequent expression of immature B-cell antigens together with stem cell antigen CD34. Blood 1992;80:470-477.

13 Porwit-MacDonald A, Janossy G, Ivory K, et al. Leukemia associated changes identified by quantitative flow cytometry. IV. CD34 overexpression in acute myelogenous leukemia M2 with t(8;21). Blood 1996; 87:1162-1169.

14 Khoury H, Dalal BI, Nevill TJ, et al. Acute myelogenous leukemia (AML) with $t(8 ; 21)$-identification of a specific immunophenotype. Leuk Lymphoma 2003;44: 1713-1718.

15 Tabernero MD, Bortoluci AM, Alaejos I, et al. Adult BALL with BCR/ABL gene rearrangement displays a unique immunophenotype based on the pattern of CD10, CD34, Cd13 and CD38 expression. Leukemia 2001;15:406-414.

16 De Zen L, Orfao A, Cazzangia G, et al. Quantitative multiparametric immunophenotyping in acute lymphoid leukemia: correlation with specific genotype. I. ETV6/AML1 ALLs identification. Leukemia 2000;14: 1225-1231.

17 Lavabre-Bertrand T, Janossy G, Ivory K, et al. Leukemia-associated changes identified by quantitative flow cytometry: I. CD10 expression. Cytometry 1994;18: 209-217.

18 Farahat N, Lens D, Zomas A, et al. Quantitative flow cytometry can distinguish between normal and leukemic B-cell precursors. $\mathrm{Br} \mathrm{J}$ Haematol 1995;91:640-646.

19 Dworzak MN, Fritsch G, Fleischer C, et al. Comparative phenotype mapping of normal vs malignant pediatric B-lymphopoiesis unveils leukemia-associated aberrations. Exp Hematol. 1998;26:305-313.

20 Lubbert M, Herrmann F, Koeffler Ph. Expression and regulation of myeloid-specific genes in normal and leukemic myeloid cells. Blood 1991;77:909-924.

21 Bennett JM, Catovsky D, Daniel MT, et al. Proposed revised criteria for classification of acute myeloid leukemia. Ann Intern Med 1985;103:621-625.

22 Mitelman F, ed. ISCN, An International System for Human Cytogenetic Nomenclature. Basel: S. Kargar, 1995.
23 Breiman L, Friedman JH, Olshen R, et al. Classification and Regression Trees. Belmont, CA: Wadsworth International Group, 1984.

24 Morton RF, Hebel JR (eds). Screening. In: A Study Guide to Epidemiology and Biostatics, 2nd edn. Gaithersburg, MD: An Aspen Publishers, 1990, pp 63-81.

25 Downing JR. The AML1-ETO chimeric protein transcription factor in acute myeloid leukemia: biology and clinical significance. Br J Haematol 1999;106:296-308.

26 Okuda T, Cai Z, Yang S, et al. Expression of knock-in AML1-ETO leukemia gene inhibits the establishment of normal definitive hematopoiesis and directly generates dysplastic hematopoietic progenitors. Blood 1998;91:3134-3143.

27 Tsuchiya H, Elsonbaty SS, Ngano K, et al. Acute myeloblastic leukemia (ANLL-M2) with t(8;21(q22;q22) variant expressing lymphoid surface antigens with high number of G-CSF receptors. Leuk Res 1993;17: 375-377.

28 Samdani A, Vijapurkar U, Grimm MA, et al. Cytogenetic and P-glycoprotein (PGP) are independent predictors of treatment outcome in acute myeloid leukemia (AML). Leuk Res 1996;20:175-180.

29 Rhoades KL, Hetherington CJ, Rowley JD, et al. Synergistic up-regulation of the myeloid-specific promoter for the macrophage colony-stimulating factor receptor by AML1 and the $t(8 ; 21)$ fusion protein may contribute to leukemogenesis. Proc Natl Acad Sci USA 1996;93:11895-11900.

30 Mitterbauer M, Kusec R, Schwarzinger I, et al. Comparison of karyotype analysis and RT-PCR for AML1/ETO in 204 unselected patients with AML. Ann Hematol 1998;76:139-143.

31 Rowe D, Cotterill SJ, Ross FM, et al. Cytogenetically cryptic AML1-ETO and CBF $\beta$-MYH11 gene rearrangements: incidence in 412 cases of acute myeloid leukemia. Br J Haematol 2000;111:1051-1056.

32 Grimwade D, Walker H, Oliver F, et al. The importance of diagnostic cytogenetics on outcome in AML: analysis of 1,612 patients entered into the MRC AML 10 trial. The Medical Research Council Adult and Children's Leukaemia Working Parties. Blood 1998; 92:2322-2333.

33 Strobl H, Takimoto M, Majidic O, et al. Myeloperoxidase expression in CD $34^{+}$normal hematopoietic cells. Blood 1993;82:2069-2078. 